Reprod. Nutr. Dévelop., 1985, 25 (1 B), 211-214.

\title{
Cellularité des différents dépôts adipeux des bovins en croissance
}

\section{J. ROBELIN}

avec la collaboration de Christiane BARBOIRON et R. JAILLER

Station des Productions Bovines et Chevalines, I.N.R.A., Theix 63122 Cevrat, France.

\section{Introduction.}

Le développement des tissus adipeux revêt une grande importance pratique chez les animaux domestiques ; ces tissus constituent en effet une réserve énergétique nécessaire durant les périodes de bilan négatif. Par ailleurs, ils déterminent en grande partie la valeur commerciale des animaux utilisés pour la production de viande. Enfin, la valeur calorique de ces tissus en fait un élément très important du coût alimentaire des animaux.

II existait peu de résultats sur le développement cellulaire des tissus adipeux des bovins jusqu'à un passé récent, en dehors des travaux de Hood et Allen (1973). Nous avons entrepris une étude descriptive de base sur le développement de ces tissus chez les bovins, du stade fœtal au stade adulte, il y a une dizaine d'années. Des résultats partiels ont déjà été publiés (Robelin, 1981). Cet article présente de façon très synthétique l'ensemble des résultats que nous avons obtenus.

\section{Matériel et méthodes.}

Nous avons mesuré par la méthode des abattages comparatifs, l'évolution du poids des principaux dépôts adipeux (après dissection complète), de leur teneur en eau et en lipides, de la taille de leurs adipocytes (après fixation par l'acide osmique). Le nombre total d'adipocytes par tissu a été calculé à partir du poids des lipides de chaque dépôt et de la taille des adipocytes. Les détails concernant ces mesures ont été publiés avec les premiers résultats de cette étude (Robelin, 1981). Ces mesures ont été réalisées sur 41 bovins mâles de race Pie-noire âgés de -20 jours à 760 jours et pesant de 32 à $680 \mathrm{~kg}$. 
Résultats - discussion.

Nous avons rapporté l'ensemble des résultats en regroupant les dépôts en trois catégories selon leur localisation : les dépôts sous-cutanés, les dépôts intermusculaires (situés entre les muscles) et les dépôts abdominaux (périrénaux, omentaux, mésentériques, péricardiaques).

Durant la période étudiée, de 20 jours avant la naissance à 760 jours après, le poids des dépôts adipeux totaux passe de $2,1 \mathrm{~kg}$ à $180 \mathrm{~kg}$ : il est multiplié par 84 (fig. 1). La croissance des dépôts sous-cutanés et abdominaux est plus rapide que celle de l'ensemble des dépôts, leur poids est multiplié par 128. Celle des dépôts intermusculaires est plus lente, leur poids étant multiplié par 57 seulement. Cette hiérarchie dans la croissance des dépôts est maintenant bien connue chez les bovins (cf. revue de Kempster, 1981).

Durant cette même période, la teneur moyenne en lipides des dépôts s'accroît de 28 à $76 \%$; ainsi le poids des lipides contenus dans l'ensemble des dépôts adipeux s'accroît de $0,6 \mathrm{~kg}$ à $137,7 \mathrm{~kg}$ : il est multiplié par 230 .

Le volume moyen des adipocytes des différents dépôts s'accroît de 3,6 à $122,0 \times 10^{-4} \mu^{3}$ : il est multiplié par 34. Le nombre total d'adipocytes des animaux passe de 1,9 à $12,4 \times 10^{10}$ : il est multiplié par 6,5 seulement. L'accroissement de la quantité de lipides stockés dans les tissus adipeux résulte donc pour une très large part de l'augmentation de la taille des adipocytes. Cependant, on observe encore un accroissement non négligeable du nombre apparent de cellules après la naissance. Ce résultat complète les premières observations de Hood et Allen (1973) et ceux de Truscott, Wood et Denny (1983) qui ne concernaient qu'une partie limitée de la vie post-natale.

Ces différentes caractéristiques sont très variables selon les dépôts (fig. 1 et 2) ; par exemple, chez les animaux de $500 \mathrm{~kg}$, les dépôts abdominaux ont une teneur en lipides plus élevée ( $84 \%$ ) que celle des dépôts sous-cutanés $(70 \%)$ ou intermusculaires $(63 \%)$. Les adipocytes des dépôts abdominaux ont un volume
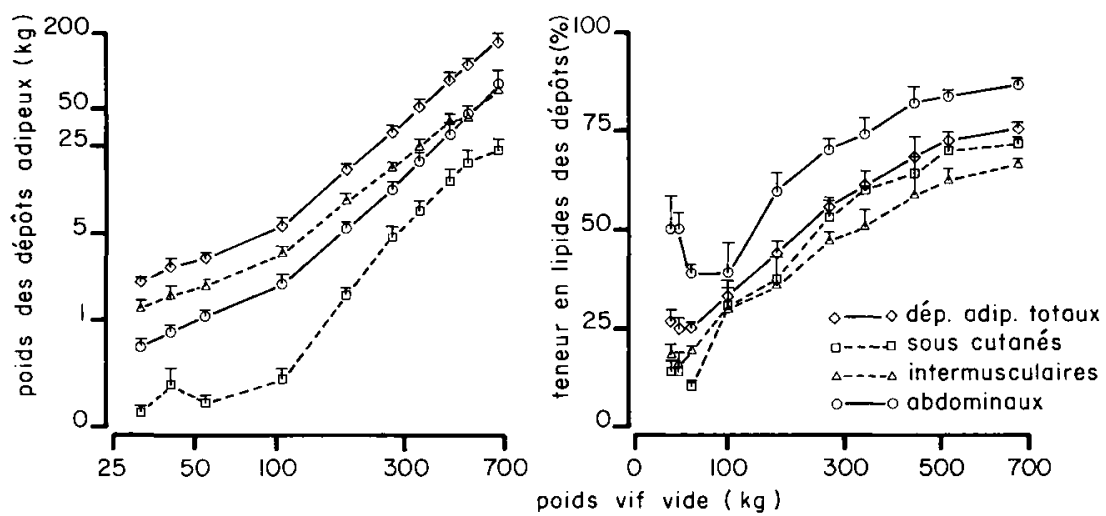

FIG. 1. - Evolution du poids et de la teneur en lipides des différems dépôts adipeux des bovins Pie-Noire au cours du développement. 
plus grand $\left(122 \times 10^{-4} \mu^{3}\right)$ que celui des cellules des autres dépôts (47 et $67 \times 10^{-4} \mu^{3}$ respectivement dans les dépôts sous-cutanés et intermusculaires).

L'augmentation de la taille moyenne des adipocytes a une origine variable dans les différents dépôts, comme le montrent les résultats présentés sur la figure 3. Dans les dépôts abdominaux, on observe tout d'abord un accroissement du nombre des cellules de diamètre inférieur à 65 microns, puis un accroissement du nombre des cellules de taille moyenne (65 à 130 microns), et enfin un accroissement du nombre des très grosses cellules. Dans les dépôts sous-cutanés, on n'observe que les deux premières phases décrites précédemment; le nombre de cellules de diamètre supérieur à 130 microns reste négligeable.

Le résultat le plus important de cette étude réside dans l'accroissement du nombre apparent de cellules chez les animaux de $300-400 \mathrm{~kg}$. Cette augmentation tardive du nombre des adipocytes observée dans différentes espèces (Nouguès et
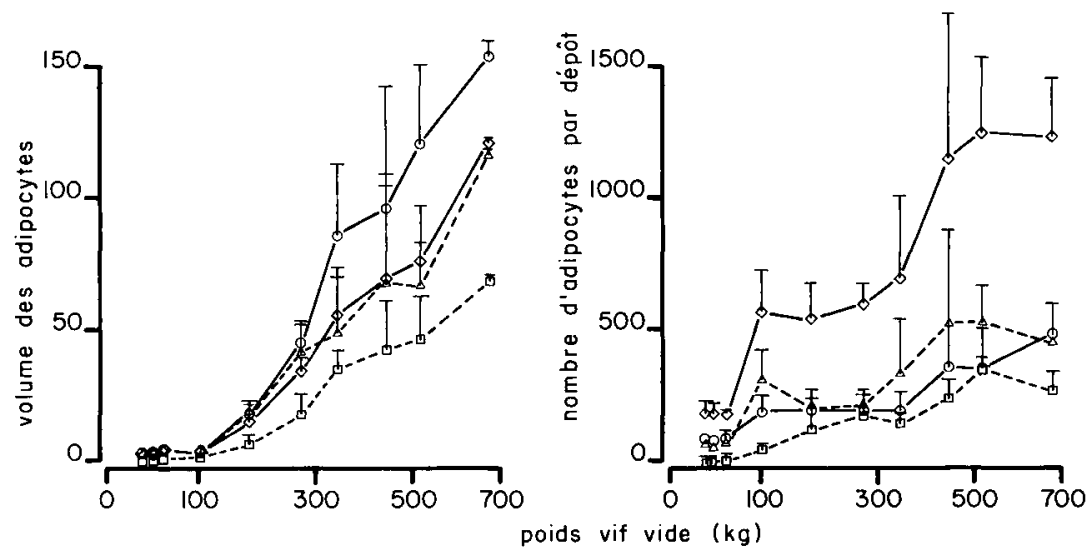

FIG. 2. - Evolution du volume $\left(10^{-4} \mu^{3}\right)$ et du nombre $\left(10^{8}\right)$ des adipocytes

des différents dépôts adipeux des bovins Pie-Noirs au cours du développement (légende = voir fig. 1).

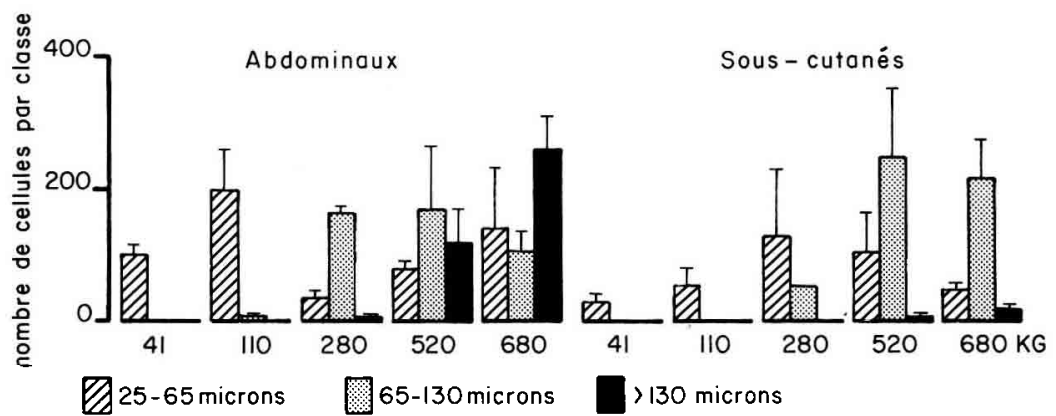

FIG. 3. - Evolution de la répartition des adipocytes dans les dépóts sous-cutanés et abdominaux des bovins Pie-Noire au cours du développement.

(Les adipocytes ont été répartis en trois classes selon leur diamètre ; on a rapporté sur la figure le nombre total d'adipocytes de chaque classe dans chacun des deux dépôts.) 
Vézinhet, 1977), résulte vraisemblablement du « recrutement » de préadipocytes ; elle semble se produire lorsque la taille moyenne des cellules atteint 100 microns. Toutefois on ne connaît ni la véritable raison de cette hyperplasie apparente, ni le signal qui pourrait la provoquer, ni le médiateur de ce signal.

Les résultats qui viennent d'être présentés sont à notre connaissance les plus détaillés parmi ceux qui existent dans l'espèce bovine. Ils permettent d'avoir une idée assez précise du développement cellulaire des dépôts adipeux et constituent une base solide pour des études ultérieures sur le métabolisme de ces tissus.

10e Réunion du groupe Développement I.N.R.A. Rennes, 9-10 mai 1984.

\section{Références}

HOOD R. L., ALLEN C. E., 1973. Cellularity of bovine adipose tissue. J. Lipid Res., 14, 605-610.

KEMPSTER A. J., 1981. Fat partition and distribution in the carcass of cattle, sheep and pigs : a review. Meat Sci., 5, 83-98.

NOUGUĖS J., VÉZINHET A., 1977. Evolution pendant la croissance de la cellularité du tissu adipeux chez le lapin et I'agneau. Ann. Biol. anim. Bioch. Biophys., 17, 799-806.

ROBELIN J., 1981. Cellularity of bovine adipose tissues: developmental changes from 15 to 65 percent of mature weight. J. Lipid Res., 22, 452-457.

TRUSCOTT T. G., WOOD J. D., DENNY H. R., 1983. Fat deposition in Hereford and Friesian steers. 2. Cellular development of the major fat depots. J. agric. Sci, 100, 271-276. 